\title{
A Transport Protocol for Future Wireless Internets
}

\author{
Deddy Chandra ${ }^{1}$ MIEEE and Richard J. Harris ${ }^{2}$ SMIEEE \\ ${ }^{1}$ School of Electrical and Computer Engineering \\ RMIT University, Melbourne, Vic, Australia \\ ${ }^{2}$ Institute of Information Sciences and Technology \\ Massey University, Palmerston North, New Zealand \\ deddy.chandra@rmit.edu.au, R.Harris@massey.ac.nz
}

\begin{abstract}
The traditional assumptions made by TCP about the operation of wired networks are often found to be invalid for wireless networks. Standard TCP semantics such as end-to-end flow control, congestion control mechanisms and error recovery provide reliability in wired networks. However, wireless communication systems have different characteristics when compared to wired networks that include higher bit error rates, higher latency, limited bandwidth, multipath fading of the signals and handoff. In this paper, we propose an enhancement to TCP that we shall call $E$ $T C P$, which improves upon conventional TCP when it is applied to the wireless environment. Our simulation results show significant improvements to TCP performance with respect to packet loss detection.
\end{abstract}

Index Terms - TCP, wireless network, congestion loss, corruption loss, handoff loss, congestion control.

\section{INTRODUCTION}

$\mathrm{T}$ HE Internet and wireless networks are arguably two of the most important technical developments that have contributed significant benefits to people's lives over the recent past. The next step in the evolution of these two developments will be convergence of the two technologies to provide widerarea wireless Internet access [16].

The Transport Control Protocol/Internet Protocol (TCP/IP) [14][16] suite is the protocol suite that integrates a wide range of different physical networks into a global Internet. TCP was developed to perform well in traditional wired networks where it serves as a transport protocol that guarantees reliable data transmission.

However, the wireless medium has different properties from those upon which the design of traditional TCP was based. In particular, TCP assumes that network links rarely corrupt data [11] and this leads to the assumption by TCP that loss of data is a signal that congestion is taking place in the network. Thus, upon the loss of a data segment, standard TCP reduces its sending rate in an attempt to alleviate perceived congestion in the network. This approach is effective if data losses are truly caused by congestion in the network. However, since wireless media have higher bit error rates leading to significant data corruption losses, such events can cause TCP to unnecessarily reduce its transmission rate, yielding a significant degradation in performance. Therefore, in order to improve TCP performance, a mechanism is required to differentiate between the possible causes of packet loss. If this can be achieved then TCP would be able to take appropriate actions according to the nature of the underlying cause of the packet loss.

Generally, there are two different approaches to improve TCP performance in a wireless network. The first approach attempts to hide any non-congestion related losses from the TCP sender and requires no changes to the existing implementation of the TCP sender. The reason is that, since the problem is local, the problem should be solved locally. As a result, most of the losses seen by the sender are due to congestion [6]. The second approach attempts to let the TCP sender become aware of the existence of a wireless environment, and consequently determine that some packet losses may not be due to congestion.

Many schemes and mechanisms have been proposed in an attempt to improve TCP performance over wireless networks. But none of them allows TCP to distinguish the cause of packet loss. It is possible to classify the schemes into three basic categories: (i) end-to-end schemes [8][10], (ii) link-layer schemes [6] and, (iii) split connection schemes [1][2].

The objective in the present work is to improve TCP performance over wireless networks. Our method falls into the second general category mentioned above, which is to make the TCP sender aware of the existence of a wireless environment and the possibility of corrupted packet losses in the connection. We propose an acknowledgment scheme together with an agent on an intermediate node that is positioned between the wired and the wireless network. This scheme allows TCP to distinguish congestion from corruption loss. Additional functionalities are required at the base station and at the TCP sender. We discuss the implementation of our proposed scheme and demonstrate its effectiveness through simulation.

The remainder of this paper is organised as follows. Section II reviews some related work in order to compare the advantages and disadvantages of existing solutions. TCP and its mechanisms are briefly described in section III. Section IV describes the assumptions concerning the inter-network structure. 
Our proposed solution is described in detail in section V. An extension to our proposed solution to overcome handoff losses will be presented in section VI. A simulation model and results will be discussed in section VII and finally, some conclusions are presented in section VIII.

\section{RELATED WORKS}

In this section we review some earlier solutions that have been published in the literature. As we shall show, most of the existing schemes do not provide any solutions for coping with handoff loss.

The Last-hop acknowledgment scheme (LHACK) was proposed in [13]. In this scheme, the base station/wireless router sends an "LHACK" ("FHACK", first-hop acknowledgment) to the stationary (mobile) source for each packet that it receives. For a connection from a fixed host to a mobile host, if "LHACK" is received at the source but the "DACK" (acknowledgment from the receiver) is missing, this infers a corruption and no congestion control mechanism is triggered. By contrast, missing both "LHACK" and "DACK" implies congestion, thus the congestion control mechanism is invoked. The drawbacks of this scheme are that two acknowledgments are sent for each message and this will result in an extra load on the return path. This scheme must rely on timeouts and fast retransmission to detect corrupted packets and it does not consider the effect of handoff for the mobile host. Significant throughput degradation occurs when multiple corruptions occur in a single window. Furthermore, for connection between two mobile hosts, the loss of "LHACK" on the wireless link to the source can be misinterpreted as network congestion.

Explicit Congestion Notification (ECN) has been discussed in [11]. The ECN scheme marks packets when it senses congestion in the router. The TCP receiver informs the sender (in a subsequent acknowledgment) about incipient congestion when a congestion marked packet has been received, which in turn triggers the congestion avoidance algorithm at the sender. The major drawback is that it requires support from both routers as well as the end hosts. This scheme introduces more processing overhead on routers and it is not feasible to do so considering there could be many routers along the path.

The Negative acknowledgment (NACK) scheme was proposed in [2]. This scheme uses the TCP cumulative acknowledgement packet with additional "NACK" in the option field to explicitly indicate which packet is received in error so that the retransmission can be initiated quickly. The drawbacks in this scheme involve its reliance on the reception of an ACK packet. If the source does not receive the ACK, it could lead to expiration of the retransmission timer. Furthermore, this scheme does not consider communication between a fixed host and a mobile host where data packets may be lost due to congestion and corruption.

Our proposed E-TCP scheme was inspired by the acknowledgement scheme known as the Explicit Loss Notification with Acknowledgment (ELN-ACK) scheme, which was proposed in [14]. Unfortunately, ELN-ACK only considers the improvement of TCP performance on a communication between a fixed sender and a mobile receiver. In contrast, their scheme does not apply to communication between the mobile sender and a fixed receiver or communication between two mobile hosts.

Caceres and Iftode [8] proposed a fast retransmission mechanism. They focussed on improving throughput and reducing interactive delay to an acceptable level by forcing TCP to retransmit segments as soon as handoff is completed, without waiting for a TCP retransmission timeout. This approach requires the transport protocol to differentiate between motion-related and congestionrelated segment loss. The advantages of this approach are that it requires modification to the end systems and relies on no special support from the underlying network or intermediate routers. The main disadvantage of fast retransmission is that it only considers host mobility (handoff) but it does not take into account the error-prone characteristics of wireless links.

\section{BACKGROUND}

\section{A. Transmission Control Protocol (TCP)}

TCP is well known as a connection-oriented transport protocol. It regulates the number of data packets that it sends by inflating and deflating a window. In order to do that TCP, on the sender side, uses the cumulative acknowledgment packets sent by the receiver. TCP has been designed with a congestion control mechanism to overcome the common problem of packet losses due to congestion in a network. The congestion control mechanism in the regular TCP (Tahoe) [12] implementation has three main parts:
a. Slow Start
b. Congestion Avoidance
c. Fast Retransmit

More details of the TCP protocol can be found in [3] and [12].

\section{B. Handoff Process}

When a mobile host is engaged in a communication, it is connected to an active base station via a radio link. If the mobile host moves to the new coverage area of another base station, the connection to the old base station is eventually disconnected, and a new 
connection to the new base station should be established to continue the communication. This process is referred to as handover or handoff.

During the handoff process, communication with the mobile host would be interrupted. Any data transmission to or from the mobile host during the handoff phase might be lost and these types of losses are known as handoff loss.

Since handoff losses are not triggered by congestion in the network, it is unnecessary for TCP to invoke its congestion control mechanism. This argument is similar to the argument for packet losses due to corruption.

\section{INTERNETWORK STRUCTURE}

We assume that the inter-network model consists of multiple networks that are joined together by computers attached to one or more networks which are known as routers. Data is delivered from a computer in one network to another computer in another network via a router. The data is forwarded from one intermediate node to another until it reaches its final destination.

As shown in Fig. 1, the inter-network may consist of a group of wired networks that form the core of a wired network and a wireless network. In general, we may assume that data are relatively corruption-free in the wired network. Thus, it is reasonable to assume that in the wired network, data might be lost due to buffer overflows, i.e. congestion. Typically, wireless networks are not used to serve as intermediate hops in the routing path between two networks. Two or more computers in the inter-network can establish a TCP session between them, regardless of whether they are mobile or fixed hosts.

We also assume that routers provide only data delivery; therefore they do not guarantee its delivery. Routers forward each data packet to the next computer or router and this machine may drop the data due to buffer overflow (congestion). Routers in wired networks are not aware of mobile hosts and must not be affected by any changes that support wireless communication. We assume that enhancing the functionality of wireless routers to the minimum level is acceptable.

Wireless routers are not responsible for the reliability of data transmission due to avoidance of processing overhead and violation of TCP semantics. Routers that are attached to wireless networks are aware that a wireless network is prone to corruption and are located at the edge of the inter-network.

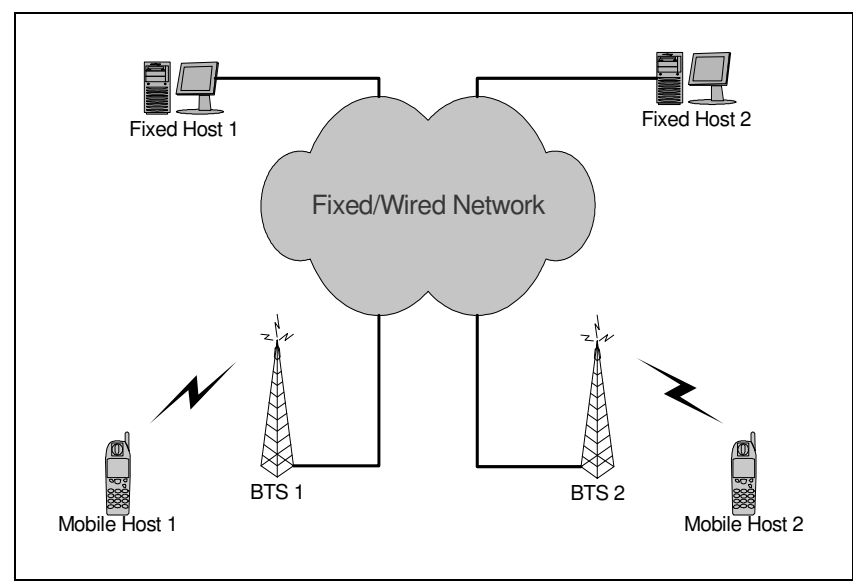

Fig. 1. Inter-networking Model

\section{ENHANCED-TCP (E-TCP)}

We propose a new enhanced TCP called E-TCP (Enhanced-TCP) that is able to differentiate packet losses due to corruption from congestion and takes appropriate action to overcome losses.

Further, we introduce the implementation of a transit agent (TA) at the base station ${ }^{1}$. A transit agent explicitly acknowledges E-TCP for the "transit history" of every packet that has transited at the base station. Thus, this acknowledgement is an indication for E-TCP to take appropriate recovery action over packet losses.

This scheme needs modifications to the structure of the acknowledgment packets and the software part of the transport protocol of the base station and the sending host. Many proposed schemes only considered communication between a fixed host and a mobile host, but our proposed scheme allows for communication between two mobile hosts as well (see Table 1).

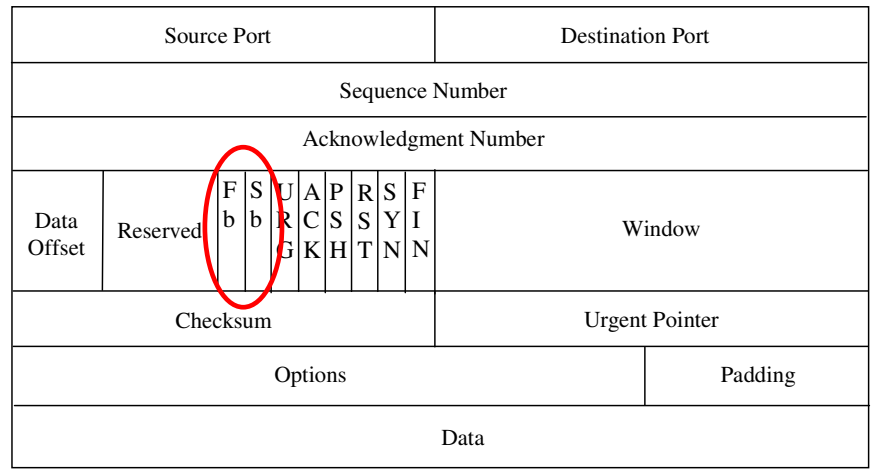

Fig. 2. Fb and $\mathrm{Sb}$ bits in the header of TCP segment

\footnotetext{
${ }^{1}$ A node that is located between the wired and wireless networks. For simplicity of understanding, we assumed here that a base station is the gateway node.
} 
TABLE 1

CROSS-COMMUNICATION BETWEEN HOSTS

\begin{tabular}{|c|c|c|}
\hline Fixed Host & TCP & E-TCP \\
\hline Mobile Host & E-TCP & E-TCP \\
\hline
\end{tabular}

\section{A. New Acknowledgment Packet}

We introduce a new form of acknowledgment packet that will be referred to as $\mathrm{ACK}_{\mathrm{C}-\mathrm{CLN}}$ (congestion-corruption loss notification). This ACK packet requires up to two additional bits in the TCP header to store information set by the transit agent. Two of the six reserved bits in the TCP header must be allocated for this purpose (see Fig. 2).

When communication occurs between a fixed host and a mobile host, data transits only one base station. Thus, only one bit is used. If communication occurs between two mobile hosts this would typically involve two base stations (Fig. 1), two bits are used instead. For simplicity, we have named each bit in the $\mathrm{ACK}_{\mathrm{C}}$ CLN packet. The first bit will be called "Fb" (First bit) while the second bit will be called "Sb" (Second bit). The first transit agent that receives an $\mathrm{ACK}_{\mathrm{C}-\mathrm{CLN}}$ packet sets the $\mathrm{Fb}$ bit and the second transit agent that receives the $\mathrm{ACK}_{\mathrm{C}-\mathrm{CLN}}$ sets the $\mathrm{Sb}$ bit.

\section{B. Transit Agent at the Base Station}

The proposed agent must be permanently allocated at the base station and it is only required to perform a simple task. The agent is required to record the sequence number of every packet that has transited through the base station before they get forwarded to the receiver. The flowchart for data processing by the transit agent is shown in Fig. 3. The agent also acknowledges the sender by providing the transit history of the expected packet through the use of $\mathrm{ACK}_{\mathrm{C}-\mathrm{CLN} \text {. }}$

The following is the data processing algorithm performed by the transit agent:

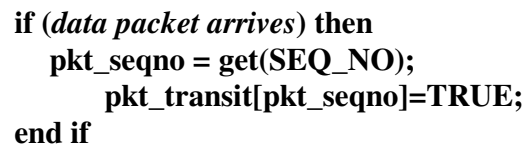

Note: initially the value of pkt_transit[pkt_id] $=$ FALSE. Where FALSE $=0$ and TRUE $=1$.

Upon receiving of an $\mathrm{ACK}_{\mathrm{C}-\mathrm{CLN}}$ packet from the receiver, the agent checks its records and sets a value for $\mathrm{ACK}_{\mathrm{C}-\mathrm{CLN}}$ as follows: $\mathrm{A}$ " 0 " in the $\mathrm{ACK}_{\mathrm{C}-\mathrm{CLN}}$ indicates the expected sequence number has never transited the base station before and a "1" indicates that the expected sequence number has transited the base station.

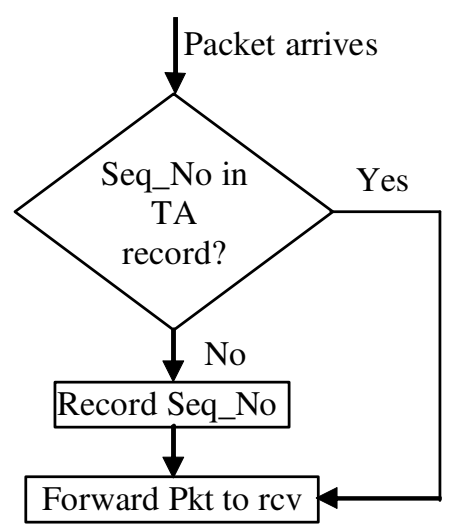

Fig. 3. Flowchart for data processing in BTS

The following is the ACK processing algorithm performed by the transit agent:

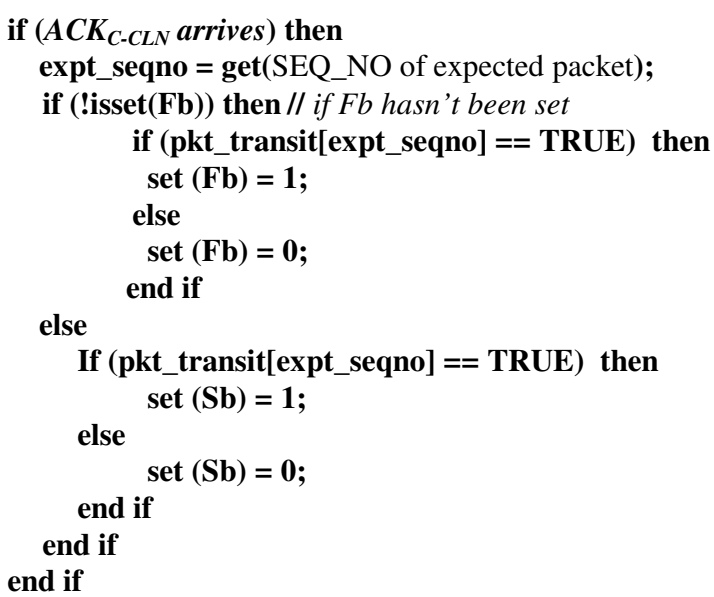

\section{Transport Protocol at Sender Side}

The E-TCP on the sender side has additional functionality to read the inserted information from the $\mathrm{ACK}_{\mathrm{C}-\mathrm{CLN}}$ packet. Thus, it takes appropriate packet loss recovery action based on the information received. The current TCP (TCP Reno) is still required for the communication between devices on the fixed network ${ }^{2}$. Therefore, the protocol stack shown in Fig. 4 is implemented on the sender side.

\begin{tabular}{|c|c|}
\hline \multicolumn{2}{|c|}{ Application } \\
\hline TCP & E-TCP \\
\hline IP \\
\hline Data Link \\
\hline Physical \\
\hline
\end{tabular}

Fig. 4. Proposed Protocol Stack

Two transport protocols are available to maintain the reliability of data transmission between the two ends, depending on the type of network node at each

\footnotetext{
${ }^{2}$ In this case the sender does not need to use E-TCP since the wireless network is not involved in data transmission and the only reason for packet loss would be network congestion
} 
end. Once the sender is able to distinguish the type of the receiver, the sender shall use TCP or E-TCP accordingly. The utilisation of both protocols is depicted in Table 1.

E-TCP will be active in the case when a wireless environment is involved in the data transmission process. An acknowledgment of the SYN packet with an $\mathrm{Fb}$ or $\mathrm{Sb}$ bit is an indication to the sender to activate the E-TCP or conventional TCP (Reno) as appropriate.

The following algorithm shows how E-TCP at the fixed sender learns about packet losses:

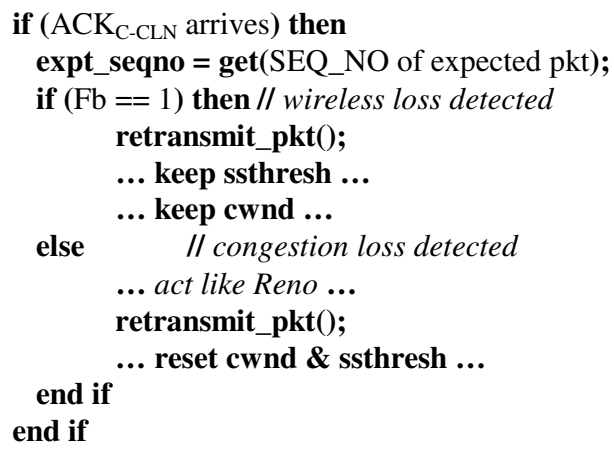

The E-TCP on the fixed sender looks simple, since it only deals with a mobile receiver. But, it will be more complicated for a mobile sender, since the receiver could be a fixed host or another mobile host. The following is the E-TCP algorithm for a mobile sender:

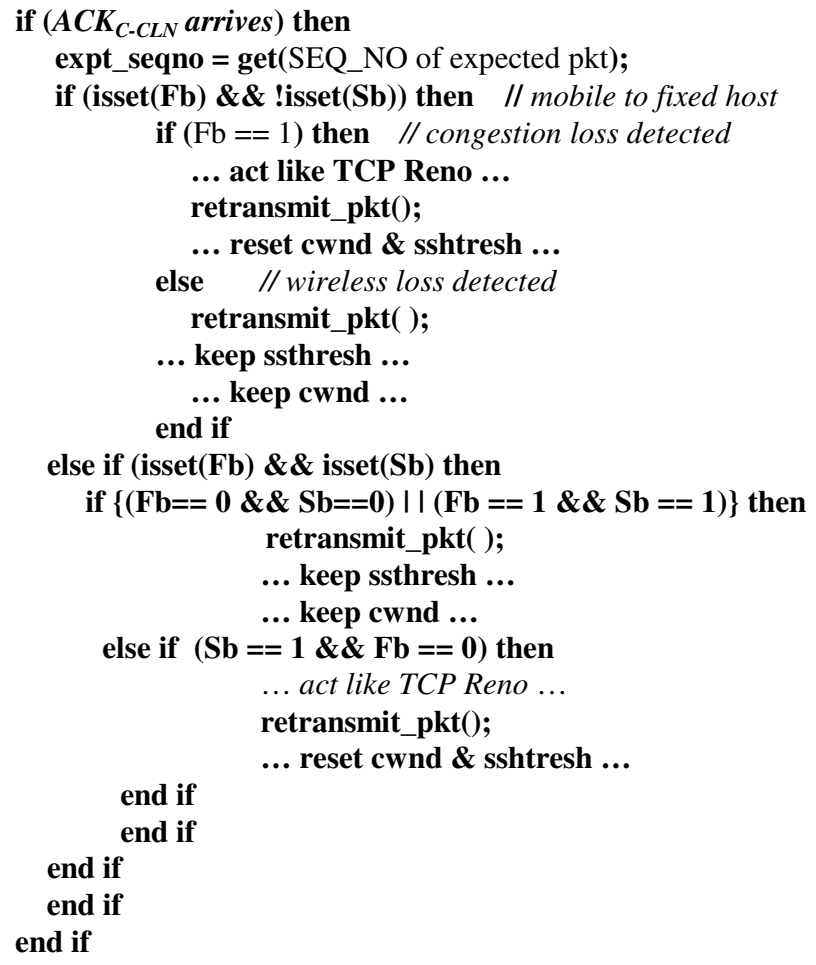

\section{Scenarios}

In our proposed protocol stack, there are four possible communication scenarios that may occur between hosts as shown in Table 1. We shall discuss all four communication scenarios below:

\section{Fixed host to fixed host}

Data transmission between hosts in a fixed network has been discussed frequently and many improvements have been suggested; therefore we leave the standard version of TCP to handle the reliability of data transmission between fixed hosts. It is unnecessary to use E-TCP in this case, since a wireless network does not involve in data transmission between fixed hosts. Therefore, any packet losses could be assumed to be as a result of congestion loss.

\section{Fixed host to mobile host}

In this scenario, a fixed sender faces the challenge of distinguishing the cause of packet loss since the data travels over both fixed and wireless networks. TCP must be able to identify the location of the packet loss in order to maintain its performance. We discover that a base station that is located right in between the two different networks is a perfect location to assist the TCP sender distinguishing the cause of packet loss. Hence, we install an agent at the base station to perform a "transit checking" task.

Each time a packet transits the base station, the transit agent records the sequence number of the packet. This record shows that the packet has safely travelled over the fixed network, transited the base station and then the packet is forwarded to the receiver.

Upon receiving an acknowledgment $\left(\mathrm{ACK}_{\mathrm{C}-\mathrm{CLN}}\right)$ packet that contains a request for the expected packet, the agent checks its records against the sequence number of that expected packet. If the agent finds the sequence number in the record, it sets the "Fb" bit to 1. Otherwise, the agent sets the "Fb" bit to 0 .

Upon receiving an $\mathrm{ACK}_{\mathrm{C}-\mathrm{CLN}}$ packet, the sender learns which packet the receiver expects. Thus, it reads the $\mathrm{Fb}$ bit, if the value equals " 1 ", the sender understands that the expected packet had transited the base station but it was not received by the receiver. This means that the expected packet was lost in the wireless network (due to corruption or, for some reason, other than congestion). On the other hand, if the value of $\mathrm{Fb}$ was " 0 ", this means the expected packet has never transited this base station before. Therefore, it responds by indicating that the packet was actually lost in the fixed network (due to network congestion).

\section{Mobile host to Fixed host}

This scenario is quite similar to the data transmission scenario between the fixed host and the mobile host. The only difference is the interpretation of the $\mathrm{Fb}$ value. When the value of $\mathrm{Fb}$ is equal to " 1 ", it means that the expected packet has previously transited the base station, thus the expected packet was lost in the fixed network. On the contrary, if the Fb 
value is equal to " 0 " then the expected packet was lost in the wireless environment.

Fig. 5 shows the flowchart of $\mathrm{ACK}_{\mathrm{C}-\mathrm{CLN}}$ processing in the base station.

\section{Mobile host to Mobile host}

Our proposed scheme also considers data transmission between mobile hosts. Since a packet must travel twice over the wireless network, the packet might transit through two base stations and travel once over a fixed network. In this case, there is the possibility of packet loss either in the wireless network or in the fixed network (Fig. 1).

In the case where packet loss occurs in the wireless area between the second base station (base station that serves the receiver) and the second mobile host (assume that this mobile host is the receiver), the sender may again assume that packet loss is due to congestion.

Therefore, the $\mathrm{ACK}_{\mathrm{C}-\mathrm{CLN}}$ must allocate another bit namely the "Sb" bit which contains the transit history of the packet set by the second transit agent. In this case, the first base station reached will set its confirmation concerning the transit status in the $\mathrm{Fb}$ bit. When this $\mathrm{ACK}_{\mathrm{C}-\mathrm{CLN}}$ reaches the second base station, the base station will assign a value for the $\mathrm{Sb}$ bit. The sender will be able to determine where the losses have occurred with information set in both the $\mathrm{Sb}$ and $\mathrm{Fb}$. Table 2 identifies the different cases of $\mathrm{Fb}$ and $\mathrm{Sb}$ associated with the type of packet loss.

TABLE 2

FB AND SB VALUES ASSOCIATED WITH THE TYPE OF PACKET LOSS CASES

\begin{tabular}{|c|c|c|}
\hline Bit & $\mathrm{Sb}=0$ & $\mathrm{Sb}=1$ \\
\hline $\mathrm{Fb}=0$ & \multirow{2}{*}{ Corruption } & Congestion \\
\cline { 1 - 1 } $\mathrm{Fb}=1$ & & Corruption \\
\hline
\end{tabular}

A packet loss that is due to corruption should be retransmitted, but it is unnecessary for the sender to reduce its transmission rate (reduce the congestion window size). More discussions concerning each case can be obtained in [3].

Fig. 6 shows the flowchart for $\mathrm{ACK}_{\mathrm{C}-\mathrm{CLN}}$ processing in the BTS for mobile-to-mobile communication.

\section{HANDOFF-RELATED PACKET LOSS}

The Enhanced-TCP (E-TCP) and Transit Agent (TA) approach was discussed in [3] and [4], has shown improvement to TCP by distinguishing the two different types of packet losses (i.e. congestion or corruption) and to invoke appropriate recovery actions accordingly. We shall now consider another type of packet loss in the wireless environment known as handoff loss.

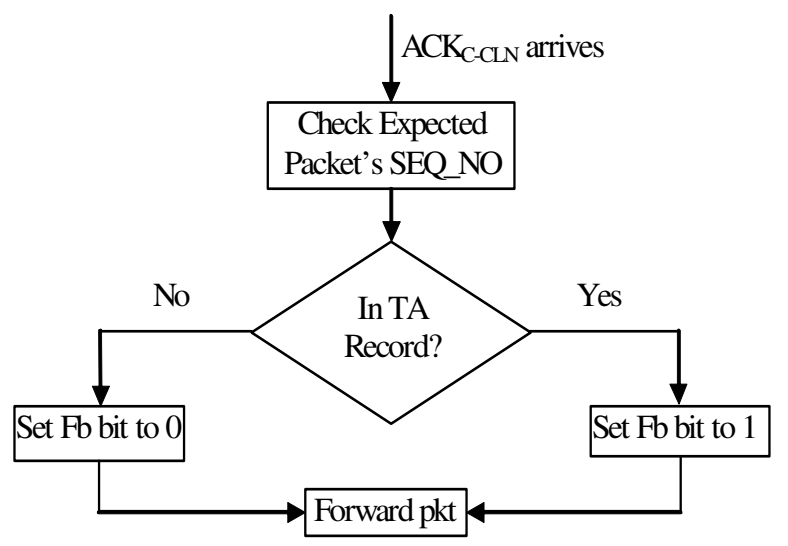

Fig. 5. Flowchart of ACKC-CLN Processing in BTS

In Fig. 7, let BTS1 represents the old base station and BTS2 represents the new base station. In the case when a data packet has been sent to a receiver through BTS1 while the mobile receiver has already been in the BTS2 coverage area, the mobile receiver may not receive the packet. Thus, the receiver will send an acknowledgment packet requesting the expected data packet. Since the TA on BTS2 does not have a transit record for the expected packet, TA will set the Fb bit to 0 . This value suggests the existence of congestion loss to the sender. In fact, this was not the case.

A handoff-related packet loss should not be overcome using the TCP congestion avoidance mechanism [3][4][7][9][14]. This is due to the fact that there was no packet loss due to congestion in the network. Therefore, handoff loss should be recovered by retransmitting the lost packet without reducing the TCP transmission rate. Instead, handoff loss should be treated in a similar way to that which E-TCP handles corruption loss. Thus, there are no changes required for the transit agent and E-TCP.

\section{A. Transit agent Synchroniser on base station controller}

Due to the limitations of transit agents that have been proposed in previous works [3][4], we have been motivated to introduce the concept of a transit agent synchronizer (TASyn), which must be positioned at the base station controller (BSC) ${ }^{3}$.

The TASyn performs a similar function to the transit agent. First, it records the sequence number of every transiting packet from the sender addressed to the mobile receiver on the currently active base station ${ }^{4}$. Second, it checks the transit history of the expected packet from its records for the old base station (assuming that the mobile host has already been in the coverage area of the new base station).

\footnotetext{
${ }^{3}$ A node that controls all the base stations, this node will have knowledge of the handoff process for mobile hosts.

4 The base station that currently handles the data transmission
} 


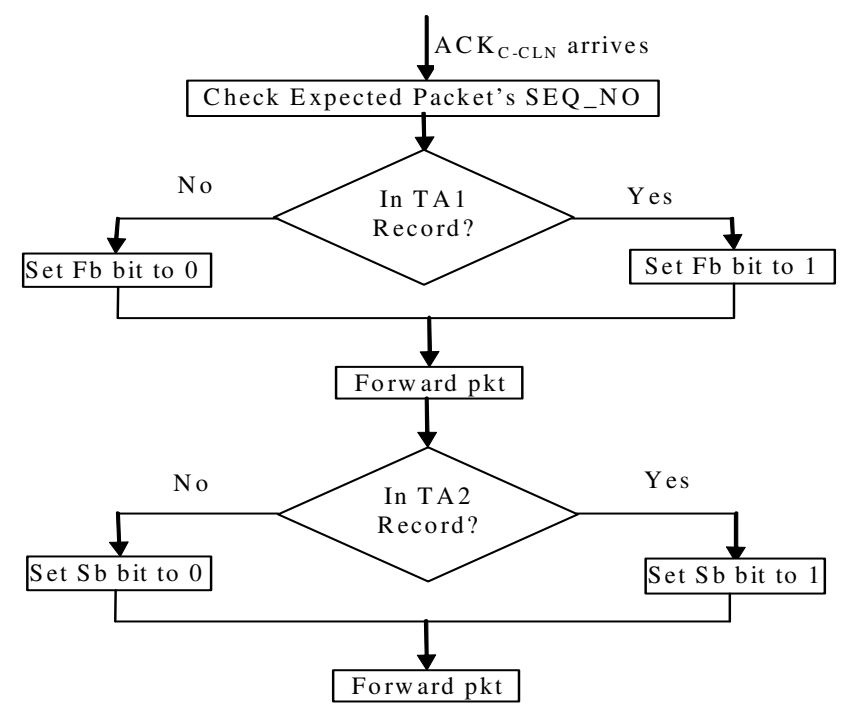

Fig. 6. Flowchart of ACKC-CLN for processing mobile-tomobile communication

If the record was found, it explains that the expected packet was lost due to handoff (since the request for the expected packet came from the receiver via the new base station).

If the TASyn has a transit record of the sequence number of the expected packet while the value of $\mathrm{Fb}$ was set to 0 by TA at the new base station, TASyn will update the $\mathrm{Fb}$ bit to 1 . The value is updated since TASyn understands that the handoff process has occurred.

The TASyn's task to update the Fb bit is over after all the handoff loss packets have been successfully retransmitted, but TASyn must continue to record the sequence numbers of any incoming data packet.

Multiple packets loss may occur during handoff process. Unfortunately, with the current implementation of TCP (Reno), it will trigger multiple reductions of the TCP congestion window size. Thus, it will significantly decrease TCP performance.

\section{SiMULATION}

This section describes a simulation study for two situations: firstly, a simulation study for E-TCP to compare it with conventional TCP in order to distinguish congestion from corruption loss; in the second case, a simulation study for E-TCP including the implementation of TASyn to overcome handoff loss is presented.

The first case was simulated in three different scenarios as depicted in Fig. 1. The sender or receiver might be a fixed host or a mobile host. The base station includes a finite-buffer drop tail gateway and the network has both wired and wireless links. It is assumed that the sender always has data to send. It is also assumed that the receiver can always send out acknowledgments immediately for each data packet received without delay, other than the processing delay.

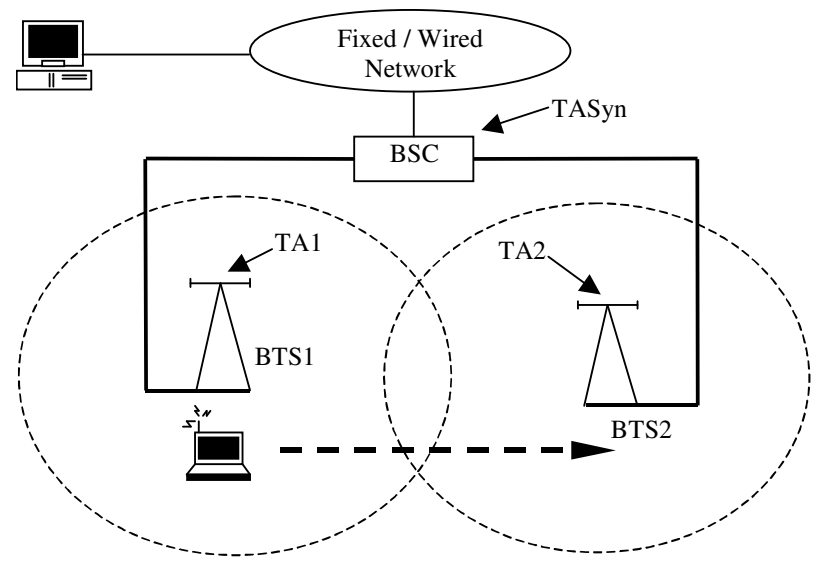

Fig. 7. Architecture for the Transit Agent Synchroniser

For simplicity, the simulations shown in this paper use a receiver that sends an ACK for every data packet received. The simulations also consist of one-way traffic. Therefore, ACK packets are never "compressed" or discarded on the path from the receiver back to the sender. The simulation models were developed with the well-known OPNET $^{\mathrm{TM}}$ simulator.

The current implementation of TCP (TCP Reno) has been considered in this simulation. The simulation was performed using a typical wireless packet loss environment and suitable bit-error rate. We assume that the bit error rate for wired links is very low.

\section{A. Congestion and Corruption Loss}

TABLE 3 summarises some parameters used in our simulation model.

TABLE 3

PARAMETER SETTINGS

\begin{tabular}{|l|l|}
\hline Wired link capacity & $56 \mathrm{Kbps}$ \\
\hline Wired link propagation delay & $30 \mathrm{msec}$ \\
\hline Wired link BER & $10^{-9}$ \\
\hline Wireless link capacity & $2 \mathrm{Mbps}$ \\
\hline Maximum window size & 94 \\
\hline TCP segment size & 170 bits \\
\hline Minimum slow-start threshold & 1 \\
\hline Bit error rate & $10^{-3}$ to $10^{-6}$ \\
\hline
\end{tabular}

In order to measure the performance of TCP over a wireless link, we simulated in all models using a range of different values for the bit-error rate (TABLE 3). All results from our simulations are obtained using standard $95 \%$ confidence levels. We have performed our simulation using three different scenarios. The three scenarios cover the following cases: We first assume that data was sent from the fixed host to the mobile host. In the second case data is transmitted from the mobile host to the fixed host and finally, data transmission from mobile to a mobile host. In all of 
these cases we assume that the mobile host stays in one cell during the lifetime of the connection. We then report the simulation results under varying bit-error conditions over the wireless channel, where the ranges of BERs are from $10^{-3}$ to $10^{-6}$.

TABLE 4

NUMBER OF RECEIVED PACKET AT RECEIVER

\begin{tabular}{|c|c|c|c|}
\hline protocol & F to M & M to F & M to M \\
\hline E-TCP & 3176 & 3341 & 3459 \\
\hline Reno-TCP & 2624 & 2722 & 2735 \\
\hline$\%$ & 21.04 & 22.74 & 26.47 \\
\hline
\end{tabular}

Performance of E-TCP and Reno on data transmission from fixed host to mobile host is depicted in Fig. 8. It shows that E-TCP performance is slightly better than Reno as the BER is increased. E-TCP allows more packets to be received. In addition, the sender was also able to maintain its transmission rate due to its new ability to distinguish between the different types of packet loss. Table 4 shows that ETCP sends, on average, about $21.04 \%$ more packets compared to Reno-TCP. The reason is that E-TCP can maintain its congestion window size. On the contrary, TCP Reno still assumes that the packet losses were due to congestion. Thus, it reduces its congestion window size (transmission rate) after the lost packet was retransmitted and the reduction in the window size results in fewer new packets being sent.

Fig. 9 shows goodput comparisons between E-TCP and TCP Reno on data transmission from mobile host to fixed host. It also shows that E-TCP can provide a better goodput than Reno. With the new functionality incorporated into E-TCP, it is able to avoid unnecessary invocation of the congestion control mechanism. On average, we see that about $22.74 \%$ more packets have been successfully received with the implementation of E-TCP.

Finally, Fig. 10 shows the goodput comparison for different values of BER whilst using E-TCP and RenoTCP for data transmission between mobile hosts. Our simulation results show that $26.47 \%$ more packets were received successfully at the receiver.

Another set of simulations was run with similar settings; except that the queue size was limited to 50 packets only. The wired link capacity was set to 100 Mbps and the wireless BER used was $10^{-3}$. Fig. 11 shows the results of a throughput comparison when Reno or E-TCP was implemented in the three different scenarios. It shows that E-TCP performance is better than Reno.

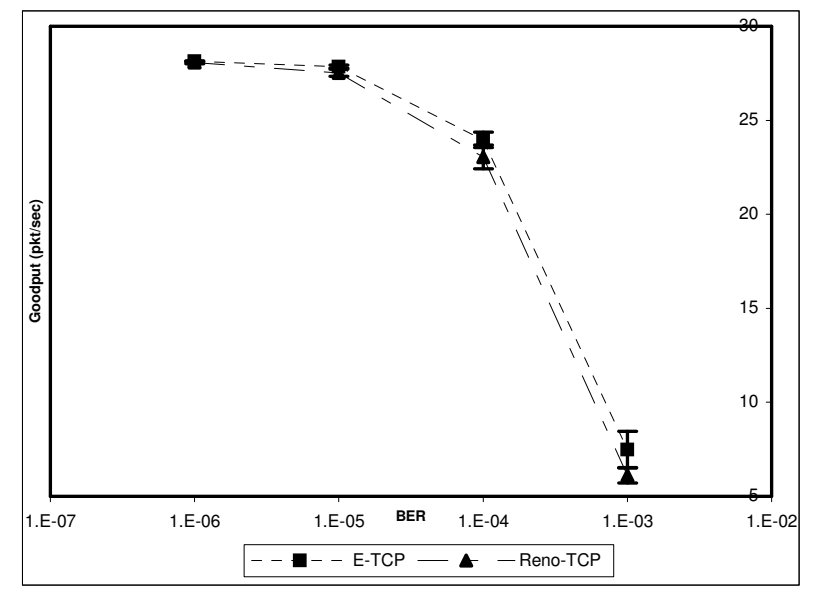

Fig. 8. Goodput comparisons at different BER on data transmission from fixed to mobile host

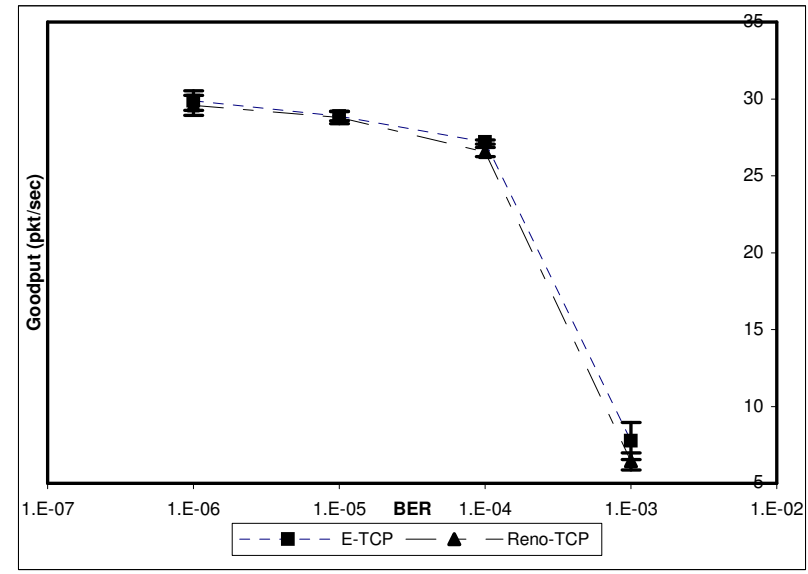

Fig. 9. Goodput comparisons at different BER on data transmission from mobile to fixed host

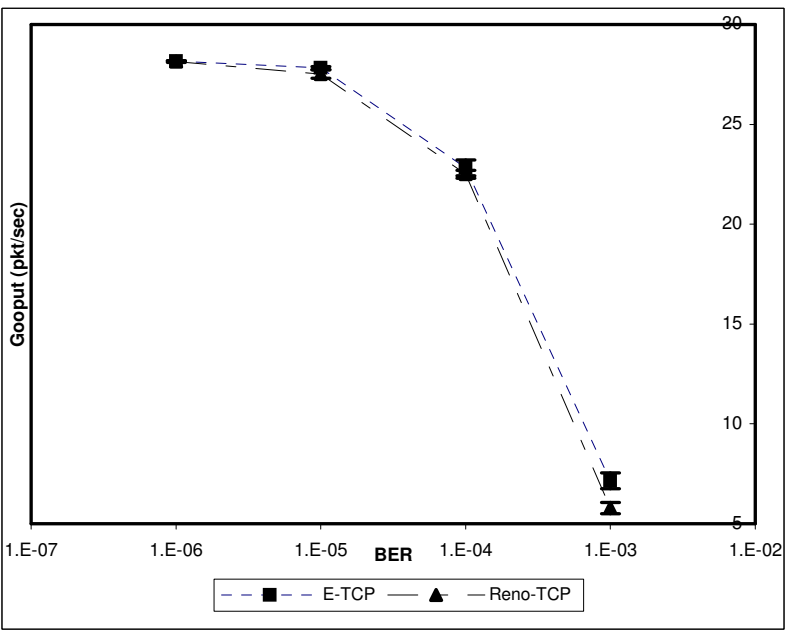

Fig. 10. Goodput comparisons at different BER on data transmissions from mobile to mobile host

Fig. 12 (a) and (b) shows the trace of congestion window size (cwnd) for E-TCP and Reno for data transmission from a fixed to a mobile host. From the figure, we can see that E-TCP can maintain a larger congestion window size compared with Reno. Since Reno is unable to distinguish the type of losses, it 
often reduces the congestion window size, which results in lower throughput.

Fig. 13 (a) and (b) shows the trace of congestion window size for E-TCP and Reno for data transmission from a mobile to a fixed host. It also shows the ability of E-TCP to maintain congestion window size for the congestion and corruption loss scenarios. On the other hand, Reno faces some difficulty in increasing its congestion window for the same scenario.

Finally, in Fig. 14 (a) and (b), we can see the trace of congestion window size for E-TCP and Reno-TCP for data transmission between mobile hosts. Reno with its ordinary congestion avoidance mechanism has been forced to reduce its congestion window size when corruption losses were detected.

\section{B. Handoff Loss}

The handoff process was simulated with the mobile host moving from one cell to another and the coverage areas were not overlapping. Some of the simulation parameters have been shown in TABLE 3. In this simulation set, the entire wired link bandwidth capacity was set to $100 \mathrm{Kbps}$. The sender in this model is a fixed host and a mobile host as the receiver.

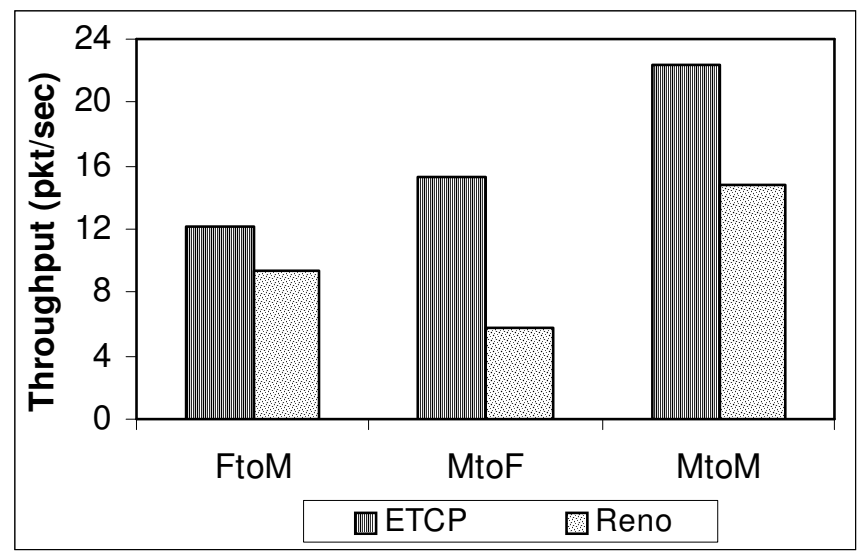

Fig. 11. Throughput comparison for the three different scenarios

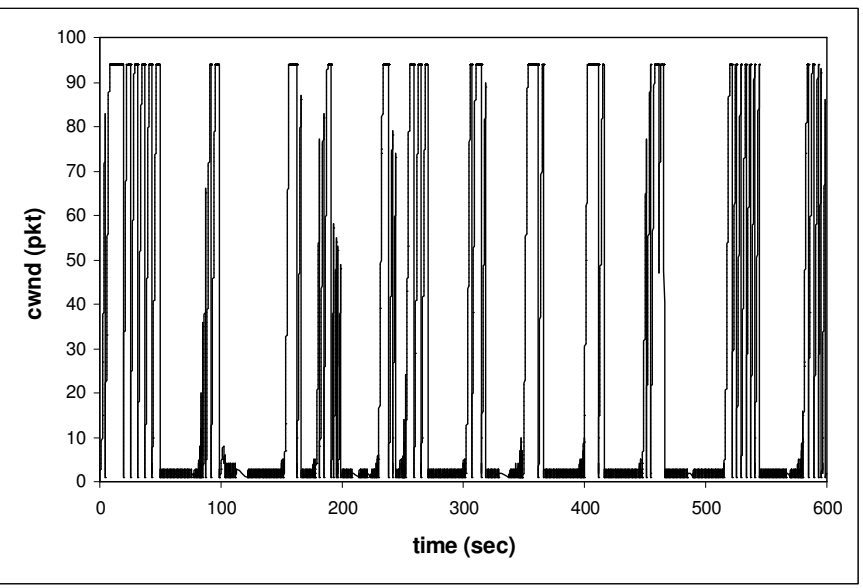

(a)

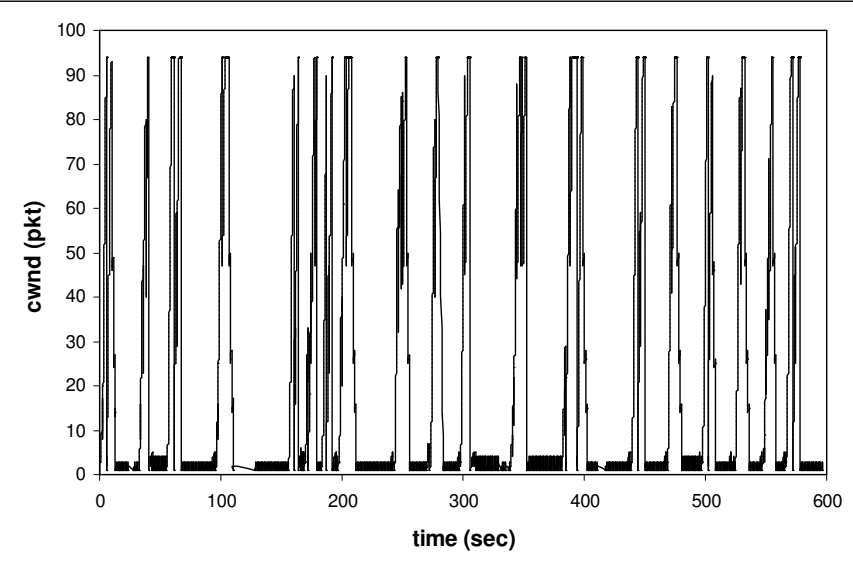

(b)

Fig. 12. Congestion window of data transmission from Fixed to Mobile host

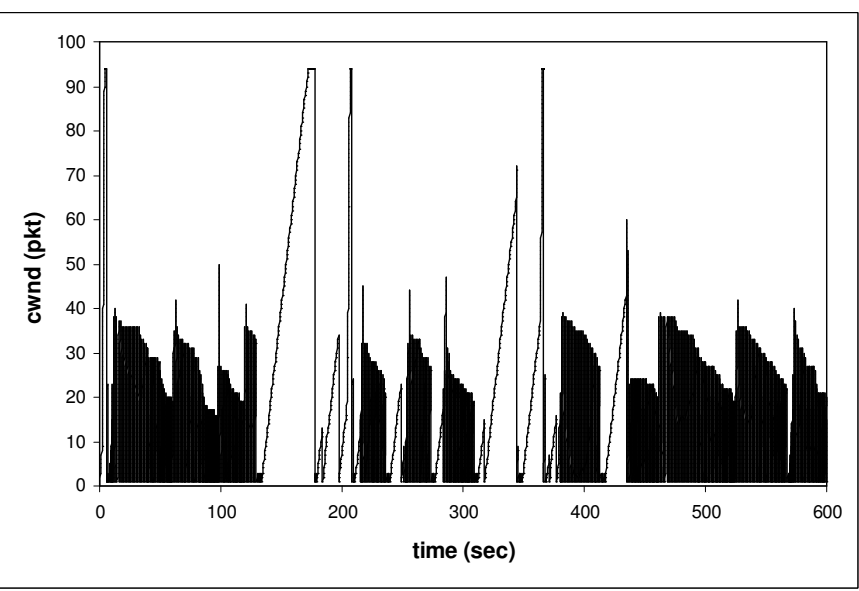

(a)

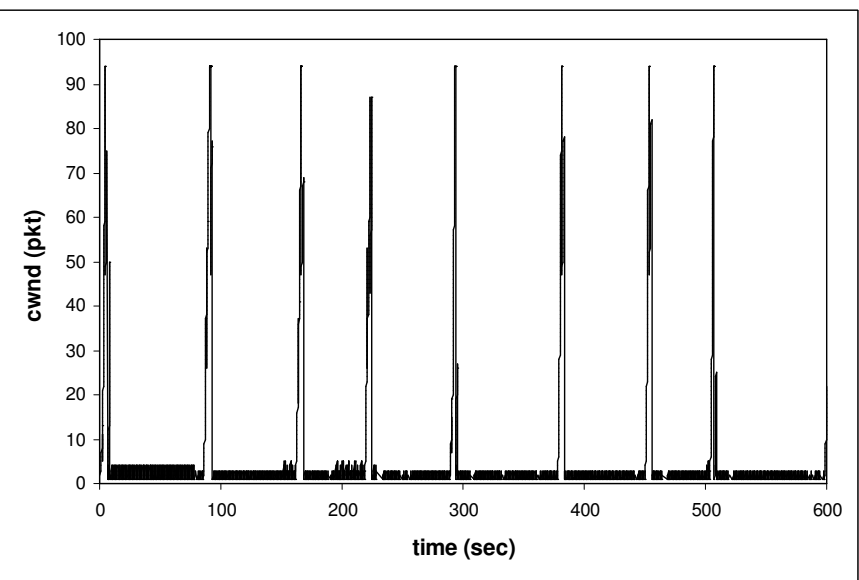

(b)

Fig. 13. Congestion window of data transmission from Mobile to Fixed host

The results from the comparison between the implementation of E-TCP with and without TASyn have been considered in this simulation. Since, this simulation set is focussing on handoff losses; the simulation model was run under an error- free wireless link and congestion-free wired link.

Fig. 15, Fig. 16 and Fig. 17 show the sender's cwnd size while the mobile receiver moves with a ground speed of 50, 100 and 150 miles/hour respectively. 
These figures focus only on the cwnd size during the handoff process.

With the implementation of TASyn, it clearly shows that E-TCP can recover faster from handoff loss. Thus, E-TCP will not suffer from a small size of cwnd (e.g. cwnd=1). The information updated by TASyn allows E-TCP to recognize handoff loss, to recover and maintain a higher transmission rate.

As in all of the following figures, it has been shown that, without implementation of TASyn, E-TCP could not keep its cwnd size and has to suffer from low cwnd for sometime until all the lost packets have been retransmitted. The retransmission of the lost packets might take a few cycles to complete and the ETCP timer might expire while retransmitting. Therefore, multiple back-off cannot be avoided and the performance of E-TCP will be low.

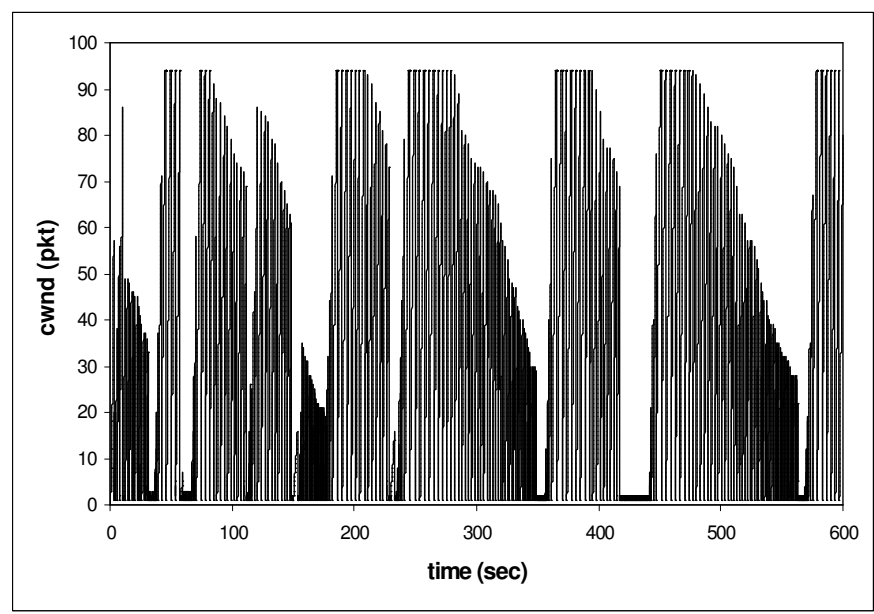

(a)

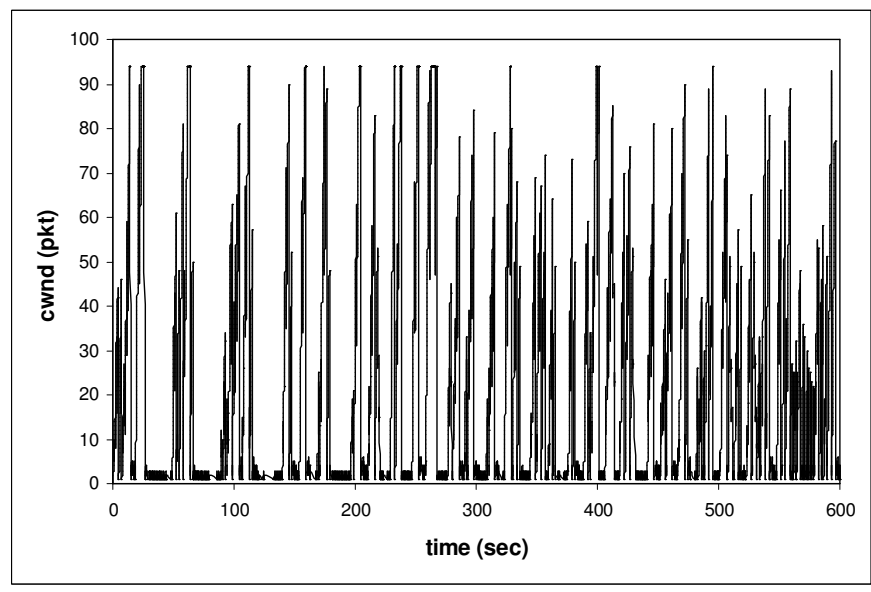

(b)

Fig. 14. Congestion window of data transmission from mobile to mobile host

As E-TCP on the sender side is able to recognise the handoff loss, it does not necessarily reduce its transmission rate and the packet loss due to handoff should be retransmitted immediately.

The implementation of E-TCP with TASyn allows better performance under the mobile handoff scenario.

\section{CONCLUSIONS}

In this paper, we have addressed problems that have been faced by TCP with the introduction of wireless networks into the Internet world. TCP Reno has no functionality to distinguish between the types of packet loss and this will cause unnecessary invocation of the congestion control mechanism to recover from these losses. As a result, unnecessary actions cause TCP to suffer from significant performance degradation.

We have proposed a novel new transport protocol called Enhanced-Transport Control Protocol (E-TCP), a new form of acknowledgment packet $\left(\mathrm{ACK}_{\mathrm{C}-\mathrm{CLN}}\right)$ and Transit Agent to solve these problems. The proposed schemes allow the transport protocol to distinguish between congestion and corruption losses. The E-TCP at the sender may adjust its transmission rate accordingly after recovery action has been taken. This scheme requires only a small amount of new functionality at the base station and at the sender, while leaving all other routers in the wired network unchanged. We have shown how to incorporate the scheme into the current implementation of the TCP protocol, and to demonstrate the effectiveness of the approach through simulation.

The key idea of the E-TCP scheme is an explicit acknowledgment scheme that enables the TCP sender to distinguish between the types of packet loss. This scheme also allows the sender to quickly discover packet loss without having to wait until the receipt of three duplicate acknowledgment packets. Therefore, the sender can recover more quickly. Since our proposed transport protocol maintains a larger congestion window size, more packets can be sent to the receiver and throughput can be maintained or increased. Simulation results show that our proposed solution provides better results compared with the implementation of TCP Reno.

Furthermore, in this paper, we have also proposed an improvement to the basic E-TCP scheme. The introduction of a transit agent synchroniser (TASyn) on a base station controller allows E-TCP to recognise handoff loss and take appropriate recovery actions.

The TASyn also requires only a small amount of functionalities while leaving E-TCP and TA unchanged. We have shown how to incorporate this additional concept into the E-TCP scheme and to demonstrate the effectiveness of the approach through simulation. 


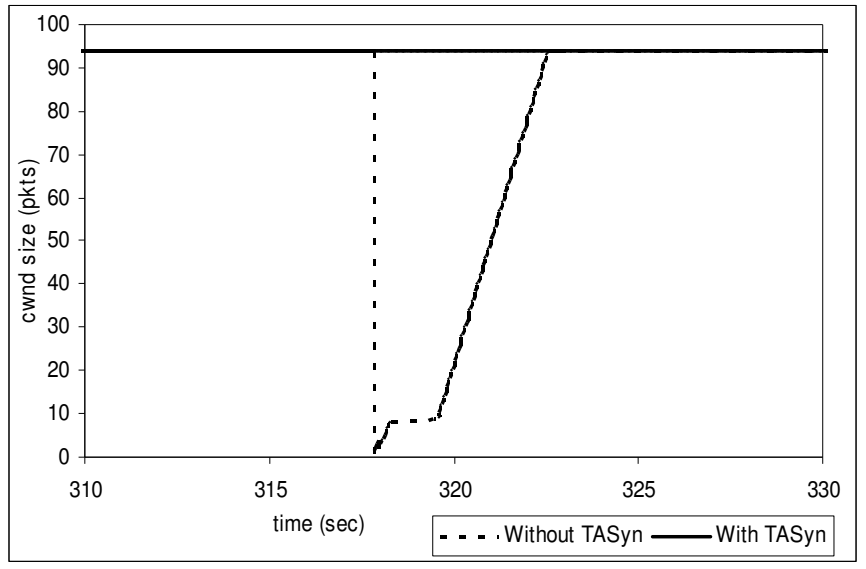

Fig. 15. The size of sender's cwnd during handoff for Ground Speed of 50 miles/hr

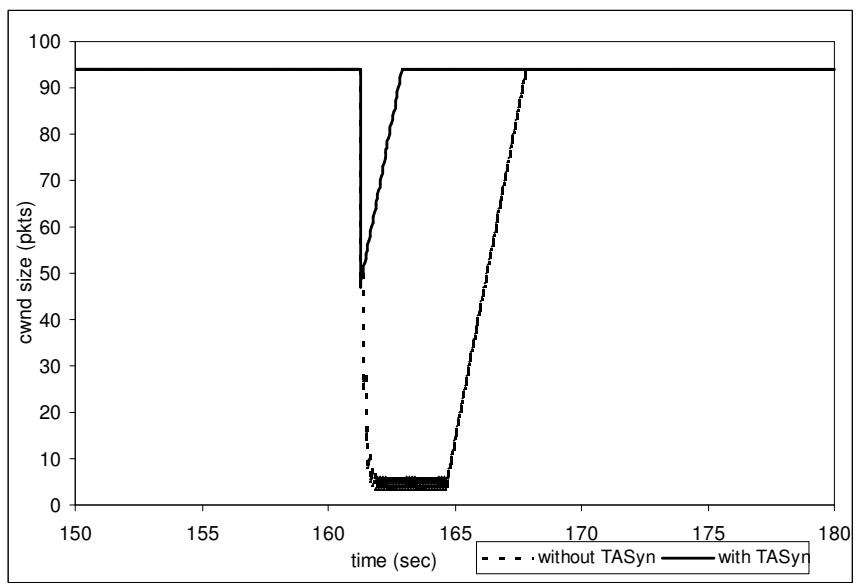

Fig. 16. The size of sender's cwnd during handoff for Ground Speed of 100 miles/hr

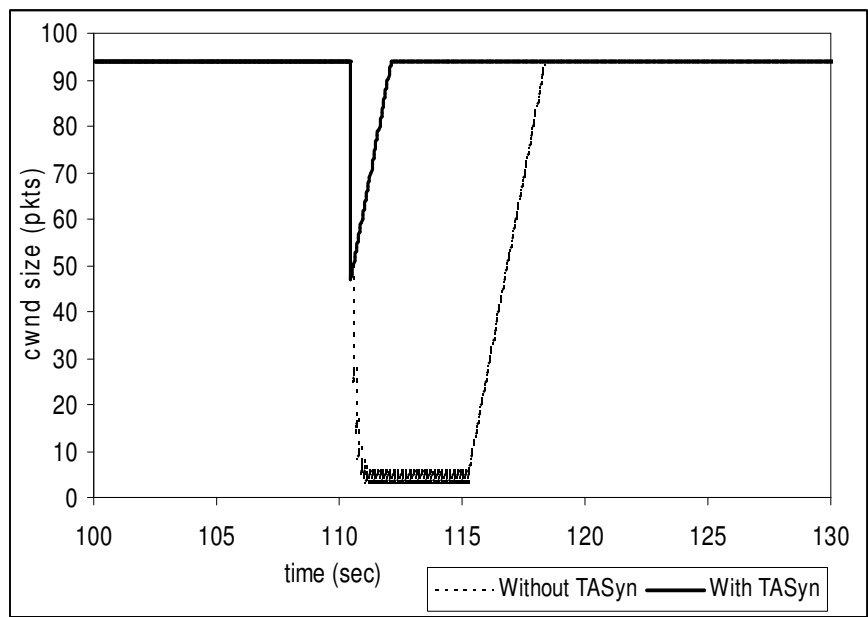

Fig. 17. The size of sender's cwnd during handoff for ground speed of $150 \mathrm{miles} / \mathrm{hr}$

\section{ACKNOWLEDGMENT}

In particular, we would like to thank the Australian Telecommunications Cooperative Research Centre (ATCRC) for their partial financial support for this paper.

\section{REFERENCES}

[1] A. Bakre and B.R. Badrinath, I-TCP:Indirect TCP for mobile hosts, in Proc. Of $15^{\text {th }}$ Int. Conf. Distributed Computing Syst. (ICDCS), May 1995.

[2] A.C.F. Chan, D.H.K Tsang and S. Gupta, "TCP (Transmission Control Protocol) over Wireless Links", In Proceeding of VTC'97, p. 2326-30, Phoenix, U.S.A., May 1997.

[3] D. Chandra, R.J. Harris and N. Shenoy, "TCP Performance for future IP-based Wireless Networks", Proceeding of $3^{\text {rd }}$ IASTED International Conference on Wireless and Optical Communications (WOC'03), pp. 521-526, July 2003.

[4] D. Chandra, R.J. Harris and N. Shenoy, "Congestion and Corruption Loss Detection with Enhanced-TCP", to appear at The Australian Telecommunication, Network and Applications Conference (ATNAC), 8-10 December 2003.

[5] D. Comer, Internetworking with TCP/IP, vol. 1-3, Reading, MA: Prentice Hall, Englewood Cliff, NJ, 1991.

[6] H. Balakrishnan, V. N Padmanabhan, S. Seshan, and R.H. Katz, A Comparison of Mechanisms for Improving TCP Performance over Wireless Links, IEEE/ACM Trans. On Networking, vol. 5, no. 6, pp. 756-769, December 1997.

[7] K. Brown and S. Singh, A Network Architecture for Mobile Computing, in Proc. of IEEE Infocom '96, pp.1388-1396, Mar 1996.

[8] L.Y. Bing, C. Imrich, "Wireless and Mobile Network Architectures", Reading, MA: John Wiley \& Sons Inc, 2001.

[9] R. Caceres, L. Iftode, Improving the Performance of Reliable Transport Protocols in Mobile Computing Environments, IEEE JSAC, vol. 13, no. 5, pp.850-857, June 1995.

[10] R.C. Durst, G.J. Miller and E.J. Travis, TCP Extension for Space Communication, Wireless Network, vol. 3, pp. 389-402, October 1997.

[11]R. Ramani and A. Karandikar, "Explicit Congestion Notification (ECN) in TCP over Wireless Network", IEEE International Conference on Personal Wireless Communications, pp. 495-499, Dec 2000.

[12] V. Jacobson, Congestion Avoidance and Control, Computer Communication Review, vol.18, no.4, pp.314-329, August 1988.

[13] J.A. Cobb, P. Agrawal, Congestion or corruption? A Strategy for Efficient Wireless TCP Sessions, IEEE Symposium on Computer and Communications (ISCC'95), June 1995.

[14] W. Ding and A. Jamalipour, "A New Loss Notification with Acknowledgment for Wireless TCP", $12^{\text {th }}$ IEEE Symposium on PIMRC, vol.1, pp. 65-69, Sep. 2001. 
[15] W.R. Stevens, TCP/IP Illustrated, Vol. 1. Reading, MA: Addison-Wesley, 1994.

[16] W. Steven, TCP Slow Start, Congestion Avoidance, Fast Retransmit, and Fast Recovery Algorithm, Internet Engineering Task Force, January 1997, RFC 2001.

[17] W.T. Chen, J.S.Lee, Some Mechanisms to Improve TCP/IP Performance over Wireless and Mobile Computing Environment, in IEEE Parallel and Distributed Systems Proceedings - Seventh International Conference, pp.437-444, July 2000.

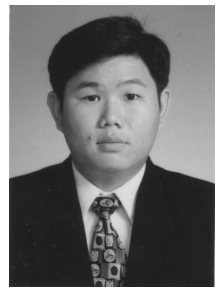

Deddy Chandra received his B.Eng in Industrial Engineering from Trisakti University, Indonesia in 1999. In 2000, he received a M.Eng degree and this was followed by a $\mathrm{PhD}$ (in the area of transport protocols for wireless networks) from the Royal Melbourne Institute of Technology (RMIT) University, Australia in 2004. His research interests include transport protocols, Internet technology, and fixed networks, wireless and mobile networks, Ad-hoc and Sensor networks. He is currently working at RMIT University in the Software and Networks Group and at Deakin University as a Research Assistant in the area of Sensor Networks.

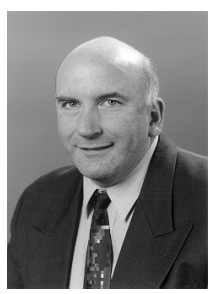

Prof. Richard Harris graduated with a B.Sc.(Hons) from the University of Adelaide in 1971 and this was followed by a Ph.D. (in the area of optimal design of telephone networks) in 1974. He moved to RMIT University in early 1993 as Director of the Centre for Advanced Technology in Telecommunications (CATT) and Professor of Communication Systems and Networks. In 2005 he moved to Massey University in New Zealand to take up a position of Chair in telecommunications and Network Engineering where he is teaching students in teletraffic engineering, communication switching, Internet engineering and conducting research in a variety of topics including network design and optimisation, network traffic management and network performance. He has participated in many consultancy projects and R \& D contracts related to Call Centre design; telephony, ATM and IP network planning, design and optimisation. He has been part of several groups involved in building network planning tools including MINDER (Telecom Australia) and network-planning tools for advanced networks such as ATM (Telekom Malaysia - APTNet) and IP network-planning for QoS. In recent years, he has been involved in the analysis of routing methods for ATM and IP networks including methods for optimal routing in OSPF and MPLS networks. 